\title{
Design of a Composite State Convergence Controller for a Nonlinear Telerobotic System
}

\author{
Muhammad Usman Asad ${ }^{1}$, Umar Farooq ${ }^{1,3}$, Jason Gu', \\ Valentina E. Balas ${ }^{2}$, Ghulam Abbas ${ }^{4}$, Marius M. Balas ${ }^{2}$ \\ ${ }^{1}$ Department of Electrical and Computer Engineering Dalhousie University, \\ Halifax, N.S. B3H 4R2, Canada \\ ${ }^{2}$ Department of Automatics and Applied Software, "Aurel Vlaicu" University of \\ Arad, Romania \\ ${ }^{3}$ Department of Electrical Engineering, University of The Punjab, Quaid-e-Azam \\ Campus, Lahore, 54590 Pakistan \\ ${ }^{4}$ Department of Electrical Engineering, The University of Lahore, Pakistan \\ mh549096@dal.ca, umar.farooq@dal.ca,jason.gu@dal.ca, \\ valentina.balas@uav.ro,ghulam.abbas@ee.uol.edu.pk,marius.balas@uav.ro
}

\begin{abstract}
This paper presents the design of a reduced complexity state convergence controller, termed as composite state convergence controller, for a single degree-offreedom nonlinear telerobotic system. First, nonlinear master and slave models are feedback-linearized and composite states are formed by combining their respective position and velocity signals. These composite master and slave states along with the operator's force are then transmitted across the communication channel instead of full states. In this way, the complexity of communication structure is reduced. An augmented system composed of composite master and slave states is finally constructed and method of state convergence is applied to compute the control gains of the proposed scheme. It is shown that position and velocity states of the master and slave systems still converge in the absence and presence of time delays, even though the design is based on the reduced order composite system. The validity of the proposed scheme is confirmed through MATLAB simulations as well as semi-real time experiments.
\end{abstract}

Keywords: state convergence; nonlinear telerobotic system; feedback linearization; composite states 


\section{Introduction}

A telerobotic system is used to perform a task in a remote location under the control of a human operator. In order to properly execute the task, feedback from the remote location is desired. The presence of feedback turns the unilateral telerobotic system into a bilateral one. This feedback can be audio, video or force signals. The force feedback can considerably improve the operator's perception of the remote environment. However, the control design of a telerobotic system involving force feedback becomes a challenging task, especially in the presence of time delays. Therefore, many research efforts have been directed to design such a robotic system [1]-[4]. The stability of a time-delayed robotic system was not guaranteed until the groundbreaking work of Anderson and Spong who employed the concepts of transmission line theory to stabilize the telerobotic system in the presence of time delays and force feedback [5]. Wave theory formalizes the transmission line concept and has become a popular framework for designing telerobotic systems [6]. The issue of wave reflection in wave-based telerobotic systems has also been addressed in the literature and improved wave-controllers have been proposed [7]. Further, more refined telerobotic systems have emerged which utilize wave variables in conjunction with neural networks [8]. The other control techniques such as $\mathrm{H}-\infty$ [9], sliding mode [10], adaptive control [11], fuzzy logic [12], disturbance-observer based control [13], etc. have also been utilized in designing telerobotic systems.

State convergence theory offers a simple and elegant way of designing the telecontrollers. This is a model-based linear control algorithm that allows the modeling of a telerobotic system on state space and requires the solution of $3 n+1$ design conditions for establishing the motion synchronization of $n^{\text {th }}$ order master and slave systems [14]. Various forms of these control algorithms are proposed in the literature. For instance, a state convergence controller with transparency condition is proposed in [15]. In another study, the state convergence controller is designed when an environment model is not available [16]. The design of nonlinear telerobotic systems based on state convergence theory has also been discussed. For instance, feedback linearization theory has been utilized to transform the nonlinear telerobotic system into a linear one and a state convergence algorithm is then employed to compute the control gains [17]. In another study, TS fuzzy model of the nonlinear telerobotic system is constructed and an appropriate parallel distributed compensation control law is designed whose gains are computed using the method of state convergence [18].

This paper is an extension of our earlier work on the channel simplification of the state convergence controller where we have only considered a linear telerobotic system [19]. The simplified architecture, termed as composite state convergence, allows transmitting fewer variables while the desired system behavior is also achieved at the same time. In this paper, we show that our reduced complexity algorithm can still be applied to a nonlinear telerobotic system. To this end, we 
first utilize the feedback linearization theory to transform the nonlinear telerobotic system into a controllable linear system. In the second stage, composite states are constructed for the transformed master and slave systems. An augmented system is then formed from the composite slave and error systems. Finally, method of state convergence is employed to compute the control gains of the proposed composite state convergence controller. In order to validate the proposed scheme, simulations are performed in a MATLAB/Simulink environment where both the delay-free and delayed communication is considered. Semi-real time experiments using the haptic device are also conducted.

This paper is structed as: Problem formulation is given in Section 2. Controller design is described in Section 3 and Results are presented in Section 4.

\section{Problem Definition}

Consider a single degree-of-freedom nonlinear teleoperation system as:

$\left\{\begin{array}{l}\text { Master: } J_{m} \ddot{\theta}_{m}+b_{m} \dot{\theta}_{m}+m_{m} g l_{m} \sin \theta_{m}=u_{m}^{\prime}+F_{m}=u_{m} \\ \text { Slave }: J_{s} \ddot{\theta}_{s}+b_{s} \dot{\theta}_{s}+m_{s} g l_{s} \sin \theta_{s}=u_{s}^{\prime}-F_{e}=u_{s}\end{array}\right.$

Where $m_{x}, l_{x}, b_{x}, J_{x}, \theta_{x}, \dot{\theta}_{x}, \ddot{\theta}_{x}, g, u_{x}$ are the mass, length, friction coefficient, inertia, angular position, angular velocity, angular acceleration, acceleration due to gravity and torque inputs for the master $(x=m)$ /slave $(x=s)$ systems, respectively. Also, $F_{m}$ and $F_{e}$ are the operator's and environment forces, respectively. By defining angular position and angular velocity as state variables i.e. $x_{1 x}=\theta_{x}, x_{2 x}=\dot{\theta}_{x}, y_{x}=x_{1 x}$, nonlinear dynamics of (1) can be written as:

$$
\begin{aligned}
& \text { Master: }\left\{\begin{array}{l}
\dot{x}_{1 m}=x_{2 m} \\
\dot{x}_{2 m}=-\frac{m_{m} g l_{m}}{J_{m}} \sin x_{1 m}-\frac{b_{m}}{J_{m}} x_{2 m}+\frac{1}{J_{m}} u_{m} \\
y_{m}=x_{1 m}
\end{array}\right. \\
& \text { Slave: }\left\{\begin{array}{l}
\dot{x}_{1 s}=x_{2 s} \\
\dot{x}_{2 s}=-\frac{m_{s} g l_{s}}{J_{s}} \sin x_{1 s}-\frac{b_{s}}{J_{s}} x_{2 s}+\frac{1}{J_{s}} u_{s} \\
y_{s}=x_{1 s}
\end{array}\right.
\end{aligned}
$$

The objective of the present study is to design control inputs for the master and slave system such that the slave is able to follow the master system and the 
environment force is also reflected to the operator as the slave interacts with the environment. Mathematically,

$$
\begin{aligned}
& \lim _{t \rightarrow \infty} x_{1 m}-\alpha x_{1 s}=0 \\
& \lim _{t \rightarrow \infty} F_{m}+\beta F_{e}=0
\end{aligned}
$$

Where $\alpha, \beta$ are scaling constants for the position and force responses, respectively. To achieve the objective in (3), we present a feedback-linearization supported composite state convergence controller in the next section.

\section{Proposed Controller}

The proposed tele-controllers for the position and force tracking task (3) are developed using the feedback linearization and composite state convergence theories. To start with, we recall the fundamentals of exact linearization.

Theorem 1: For a nonlinear system $\dot{x}=f(x)+g(x) u, y=h(x)$ having a relative degree $n$ for all $x \in \square^{n}$, there exists a transformation $\phi(x)$ such that the resulting system $\dot{z}=A z+B v$ is linear and controllable in new coordinates. The coordinate transform, nonlinear input and the resuting linear system are given as:

$$
\begin{aligned}
& z=\phi(x)=\left[\begin{array}{c}
z_{1} \\
z_{2} \\
\vdots \\
z_{n}
\end{array}\right]=\left[\begin{array}{c}
\phi_{1}(x) \\
\phi_{2}(x) \\
\vdots \\
\phi_{n}(x)
\end{array}\right]=\left[\begin{array}{c}
h(x) \\
L_{f} h(x) \\
\vdots \\
L_{f}^{n-1} h(x)
\end{array}\right] \\
& u=\frac{1}{L_{g} L_{f}^{n-1} h(x)}\left(-L_{f}^{n} h(x)+v\right) \\
& \dot{z}=\left[\begin{array}{c}
\dot{z}_{1} \\
\dot{z}_{2} \\
\vdots \\
\dot{z}_{n}
\end{array}\right]=\left[\begin{array}{ccccc}
0 & 1 & 0 & \ldots & 0 \\
0 & 0 & 1 & \ldots & 0 \\
0 & 0 & 0 & \ldots & 1 \\
0 & 0 & 0 & \ldots & 0
\end{array}\right]\left[\begin{array}{c}
z_{1} \\
z_{2} \\
\vdots \\
z_{n}
\end{array}\right]+\left[\begin{array}{c}
0 \\
0 \\
\vdots \\
1
\end{array}\right] v
\end{aligned}
$$

Where $L_{f} h(x)$ is lie-derivative of $h(x)$ in the direction of $f(x)$ and is determined as $L_{f} h(x)=\sum_{i=1}^{n} \frac{\partial h}{\partial x_{i}} f_{i}(x)$. 
The application of Theorem 1 on the nonlinear master and slave models in (2) yields the following linearized tele-robotic system:

$$
\begin{gathered}
\text { Master }:\left\{\begin{array}{l}
\phi_{m}=\left[\begin{array}{ll}
x_{1 m} & x_{2 m}
\end{array}\right]^{T} \\
\dot{z}_{1 m}=z_{2 m} \\
\dot{z}_{2 m}=v_{m} \\
u_{m}=b_{m} x_{2 m}+m_{m} g l_{m} \sin x_{1 m}+J_{m} v_{m} \\
y_{m}=x_{1 m}=z_{1 m}
\end{array}\right. \\
\text { Slave : }\left\{\begin{array}{l}
\phi_{s}=\left[\begin{array}{ll}
x_{1 s} & x_{2 s}
\end{array}\right]^{T} \\
\dot{z}_{1 s}=z_{2 s} \\
\dot{z}_{2 s}=v_{s} \\
u_{s}=b_{s} x_{2 s}+m_{s} g l_{s} \sin x_{1 s}+J_{s} v_{s} \\
y_{s}=x_{1 s}=z_{1 s}
\end{array}\right.
\end{gathered}
$$

After the master and slave systems are exactly linearized through (5), communication between them is established using the composite state convergence methodology proposed by the authors. The overall control scheme is shown in Fig. 1. We now show the convergence of master and slave systems' states as well as force reflection ability of the proposed scheme.

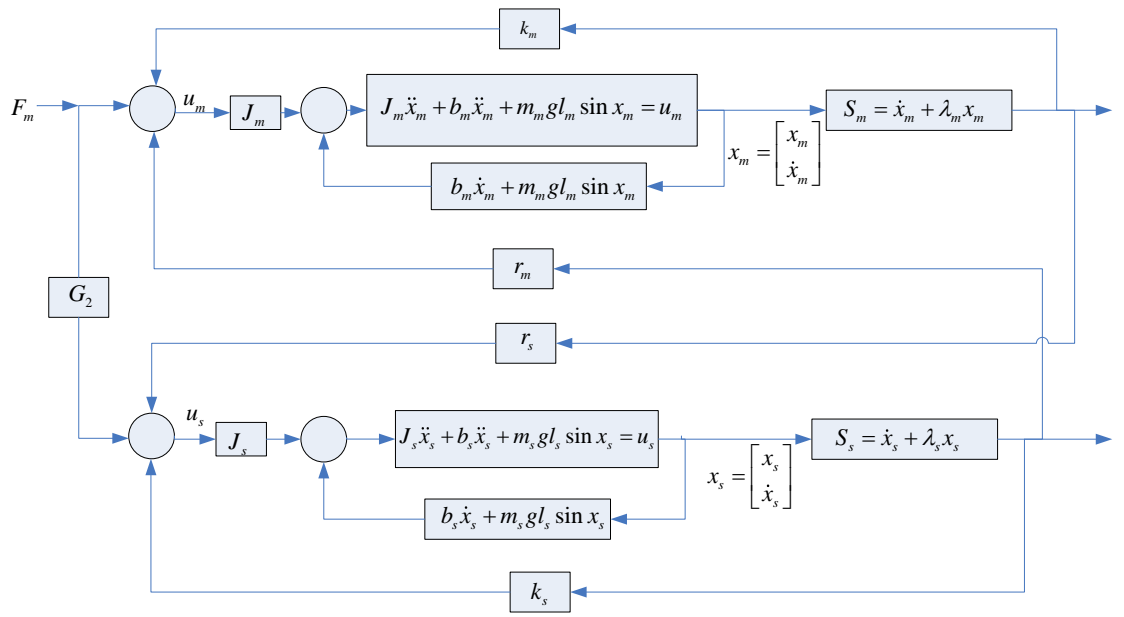

Figure 1

Proposed Scheme

Theorem 2: The slave system is able to follow the master system in the absence of communication time delay if gains of the composite state convergence controller are found as a solution of the following design conditions: 


$$
\begin{aligned}
& G_{2}-1=0 \\
& k_{s}+r_{s}-k_{m}-r_{m}=0 \\
& k_{s}+r_{s}=-p \\
& k_{m}-r_{s}=-q
\end{aligned}
$$

Proof: Let us define the composite states for the master $\left(s_{m}\right)$ and slave $\left(s_{s}\right)$ systems as:

$$
\begin{aligned}
& s_{m}=x_{2 m}+\lambda_{m} x_{m 1} \\
& s_{s}=x_{2 s}+\lambda_{s} x_{1 s}
\end{aligned}
$$

The time derivative of (10) along with (5) yields the composite dynamical system as:

$$
\begin{aligned}
& \dot{s}_{m}=v_{m}+\lambda_{m} x_{m 2} \\
& \dot{s}_{s}=v_{s}+\lambda_{s} x_{s 2}
\end{aligned}
$$

Let us define the control inputs for the feedback-linearized tele-robotic system as:

$$
\begin{aligned}
& v_{m}=-\lambda_{m} x_{m 2}+k_{m} s_{m}+r_{m} s_{s}+F_{m} \\
& v_{s}=-\lambda_{s} x_{s 2}+k_{s} s_{s}+r_{s} s_{m}+G_{2} F_{m}
\end{aligned}
$$

By plugging (12) in (11), we get:

$$
\begin{aligned}
& \dot{s}_{m}=k_{m} s_{m}+r_{m} s_{s}+F_{m} \\
& \dot{s}_{s}=k_{s} s_{s}+r_{s} s_{m}+G_{2} F_{m}
\end{aligned}
$$

Let $s_{e}=s_{s}-s_{m}$ be the composite error. The composite error dynamics can be written using (13) as:

$$
\dot{s}_{e}=\left(k_{s}+r_{s}-k_{m}-r_{m}\right) s_{s}+\left(k_{m}-r_{s}\right) s_{e}+\left(G_{2}-1\right) F_{m}
$$

We now form an augmented system comprising of composite slave and error systems as:

$$
\left[\begin{array}{c}
\dot{s}_{s} \\
\dot{s}_{e}
\end{array}\right]=\left[\begin{array}{cc}
k_{s}+r_{s} & -r_{s} \\
k_{s}+r_{s}-k_{m}-r_{m} & k_{m}-r_{s}
\end{array}\right]\left[\begin{array}{l}
s_{s} \\
s_{e}
\end{array}\right]+\left[\begin{array}{c}
G_{2} \\
G_{2}-1
\end{array}\right] F_{m}
$$

We now allow the composite error to evolve as an autonomous system, which yields the design conditions (6) and (7). The charateristic equation of the remaining augmented system is finally compared with the desired polynomial $(s+p)(s+q)=0$ which yields the design condition (8) and (9). Now, it is left to show that states of the slave system converge to the states of the master system with the control gains in (6)-(9). These control gains yield the closed loop master 
as well as slave system as $\ddot{x}_{1 z}+\left(\lambda_{z}+p\right) \dot{x}_{1 z}+\lambda_{z} p=F_{m}$ which implies that slave position can be made to track the master position with the scaling factor as $\alpha=\lambda_{m} / \lambda_{s}$ which also implies the zero convergence of the velocity states. This completes the proof.

Theorem 3: The motion of the slave system will be synchronized with the master system in the presence of communication time delay ( $\mathrm{T}$ ) if control gains of the composite nonlinear controller are found as a solution of the following design conditions:

$$
\begin{aligned}
& G_{2}\left(1+T r_{m}\right)-T r_{s}=1 \\
& k_{s}+\left(1-T k_{m}\right) r_{s}-k_{m}+\left(T k_{s}-1\right) r_{m}=0 \\
& k_{s}-\operatorname{Tr}_{s} r_{m}+r_{s}-\operatorname{Tr}_{s} k_{m}=-p \\
& r_{s}-\operatorname{Tr}_{s} k_{m}-r_{m}+\operatorname{Tr}_{m} k_{s}=-q
\end{aligned}
$$

Proof: Consider the tele-robotic system of Fig. 1 with time delay, $\mathrm{T}$ in the communication paths. Let the virtual inputs for the master and slave systems be introduced as:

$$
\begin{aligned}
& v_{m}=-\lambda_{m} x_{m 2}+k_{m} s_{m}+r_{m} s_{s}(t-T)+F_{m} \\
& v_{s}=-\lambda_{s} x_{s 2}+k_{s} s_{s}+r_{s} s_{m}(t-T)+G_{2} F_{m}(t-T)
\end{aligned}
$$

The delayed dynamical composite master and slave systems can be derived as:

$$
\begin{aligned}
& \dot{s}_{m}=k_{m} s_{m}+r_{m} s_{s}(t-T)+F_{m} \\
& \dot{s}_{s}=k_{s} s_{s}+r_{s} s_{m}(t-T)+G_{2} F_{m}(t-T)
\end{aligned}
$$

Let us now use the first order Taylor series expansion on the time delayed signals with the assumption of constant operator force, i.e.

$$
\begin{aligned}
& s_{x}(t-T) \approx s_{x}-T \dot{s}_{x}, x=m, s \\
& F_{m}(t-T) \approx F_{m}-T \dot{F}_{m}=F_{m}
\end{aligned}
$$

Based on the above Taylor expansion and using the definition of composite error, the closed loop delayed composite master and slave systems can be written as:

$$
\begin{aligned}
& \dot{s}_{m}=\frac{1}{\left(1-T^{2} r_{s} r_{m}\right)}\left(\left(k_{m}-\operatorname{Tr}_{s} r_{m}+r_{m}-T r_{m} k_{s}\right) s_{s}-\left(r_{m}-T r_{m} k_{s}\right) s_{e}+\left(1-T r_{m} G_{2}\right) F_{m}\right) \\
& \dot{s}_{s}=\frac{1}{\left(1-T^{2} r_{s} r_{m}\right)}\left(\left(k_{s}-\operatorname{Tr}_{s} r_{m}+r_{s}-\operatorname{Tr}_{s} k_{m}\right) s_{s}-\left(r_{s}-T r_{s} k_{m}\right) s_{e}+\left(G_{2}-T r_{s}\right) F_{m}\right)
\end{aligned}
$$


We now write the composite slave-error augmented system:

$$
\left.\left.\left[\begin{array}{c}
\dot{s}_{s} \\
\dot{s}_{e}
\end{array}\right]=\frac{1}{\left(1-T^{2} r_{s} r_{m}\right)}\left(\left[\begin{array}{ll}
k_{s}-\operatorname{Tr}_{s} r_{m}+r_{s}-T r_{s} k_{m} & -r_{s}+T r_{s} k_{m} \\
\left(k_{s}+r_{s}-T r_{s} k_{m}-k_{m}-\right. \\
r_{m}+T r_{m} k_{s}
\end{array}\right)\left(\begin{array}{l}
r_{s}-T r_{s} k_{m}- \\
r_{m}+T r_{m} k_{s}
\end{array}\right)\right]\left[\begin{array}{l}
s_{s} \\
s_{e}
\end{array}\right]+\left[\begin{array}{c}
G_{2}-T r_{s} \\
\left(G_{2}-T r_{s}-1\right. \\
+T r_{m} G_{2}
\end{array}\right)\right] F_{m}\right)
$$

The composite error system is now allowed to evolve as an autonomous system which leads to the design conditions (16), (17). The rest of the augmented system is then assigned the desired dynamics formed from the poles $s=-p, s=-q$. This assignment leads to the design conditions (18), (19). An analysis similar to Theorem 2 reveals that the slave system indeed follows the master system. The proof is now completed.

\section{Results \& Discussion}

The proposed composite nonlinear state convergence controller is simulated in MATLAB/Simulink environment to evaluate its effectiveness in motion synchronization of master and slave systems. For the purpose of simulations, parameters of the tele-robotic system are adopted from [17]:

$$
\begin{aligned}
& \text { Master }: m_{m}=1, l_{m}=0.2, b_{m}=10, J_{m}=0.33 m_{m} l_{m}^{2} \\
& \text { Slave }: m_{s}=10, l_{s}=1, b_{s}=15, J_{s}=0.33 m_{s} l_{s}^{2} \\
& \text { Environment }: k_{e}=10, k_{f}=1
\end{aligned}
$$

We first perform simulations when no time delay exists in the communication channel. To this end, let the desired poles be placed at $s+p=s+2, s+q=s+20$ and let the motion scaling constants be selected as unity. The design conditions in Theorem 1 are then solved and following control gains are obtained:

$$
\begin{aligned}
& G_{2}=1 \\
& k_{m}=-12 \\
& k_{s}=-10 \\
& r_{s}=8
\end{aligned}
$$

By assuming zero initial conditions for both the master and slave systems, telerobotic system is simulated under a constant operator's force of $0.2 \mathrm{~N}$ and the control gains of (28). The result is depicted in Figs. 2-3. It can be seen that the composite states convergence and this leads to the convergence of master and slave systems' states. The motion scaling property of the proposed controller is 
also evaluated in simulations. Let it be desired that slave's motion converges to $50 \%$ of the motion of the master system which leads to the selection of slave's scaling constant as $\lambda_{s}=2$. The simulations are now run with the control gains of (28) and the result is shown in Fig. 4. It can be seen that the slave's position response is indeed 0.5 times the position profile of the master system.

We now test the proposed controller when time delay exists in the communication channel. Let the time delay be $0.2 \mathrm{~s}$ in each direction. With the parameters of the tele-robotic system in (27) and using the same desired dynamics as in the delayfree case, control gains are found based on the design conditions of Theorem 3 as:

$G_{2}=0.3521$

$k_{m}=-16$

$k_{s}=-2.3944$

$r_{s}=0.2817$

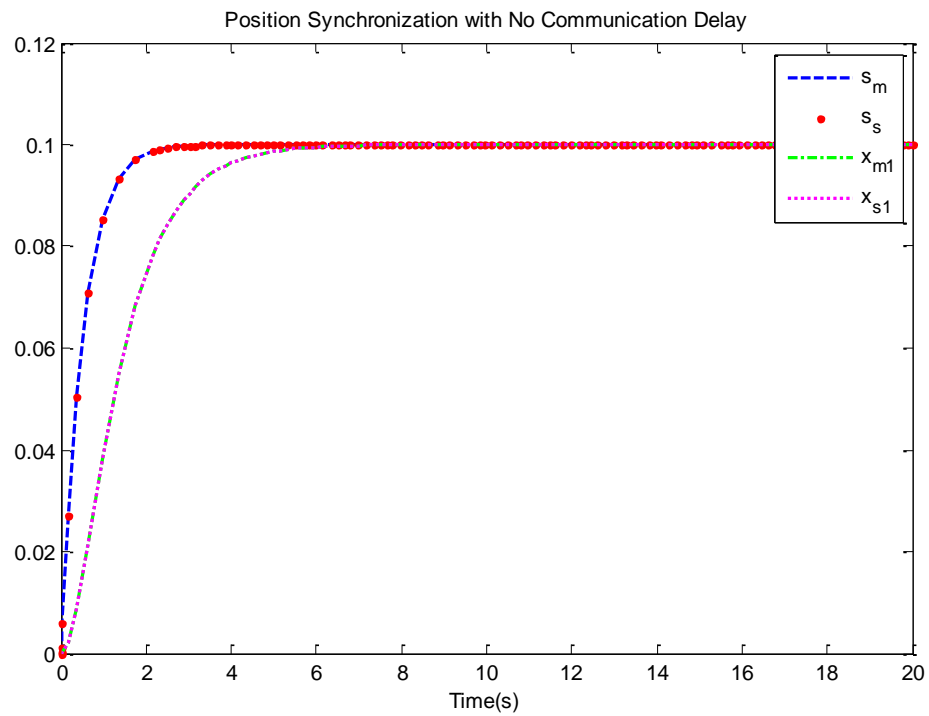

Figure 2

Position synchronization with no communication time delay 


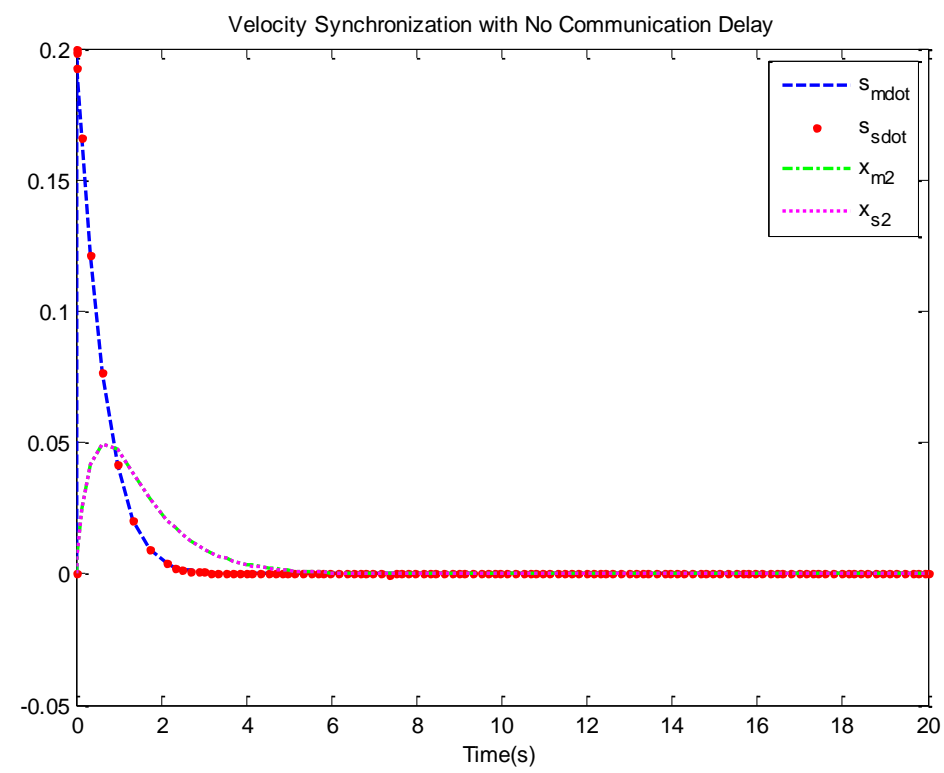

Figure 3

Velocity synchronization with no communication time delay

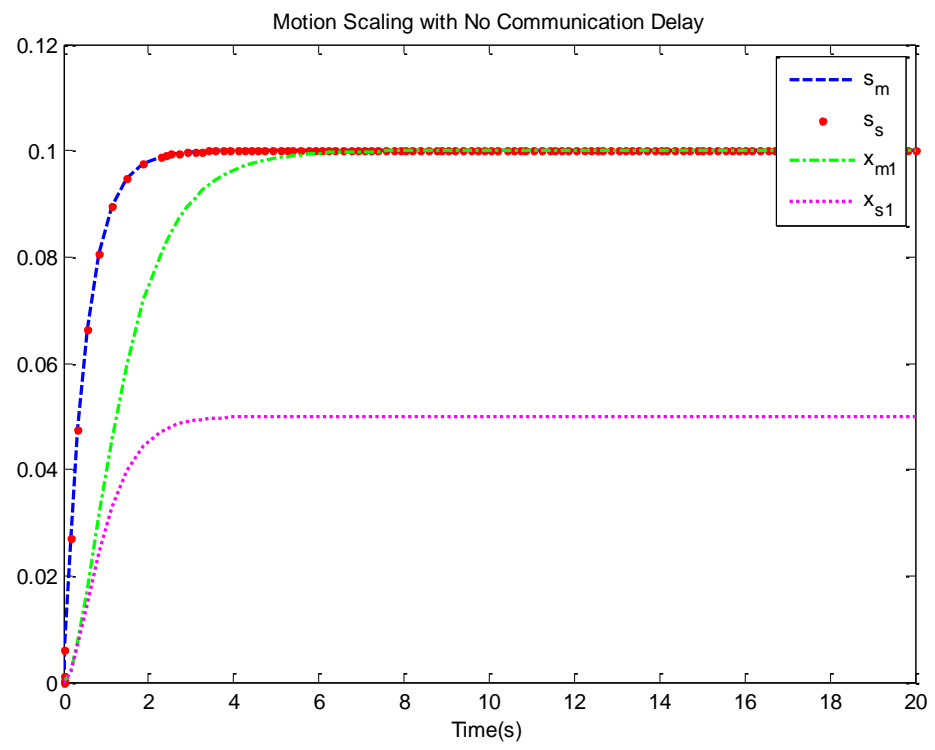

Figure 4

Motion scaling with no communication time delay 
By selecting $x_{m}(0)=x_{s}(0)=0, \lambda_{m}=\lambda_{s}=1$ and with a constant operator's force of $0.2 \mathrm{~N}$, we run the time-delayed tele-robotic system under the control gains of (29) and the results are shown in Figs. 5-6. The analysis reveals that the composite slave system follows the composite master system which leads to convergence of the position and velocity states of the master and slave systems. The motion scaling ability of the time-delayed tele-robotic system is also investigated. To this end, reference for the slave system is set as $0.25 x_{m 1}$ which implies $\lambda_{s}=4$. The simulation result, as obtained under the control gains of (29), is shown in Fig. 7. It can be seen that the motion of the slave system has been achieved as desired.

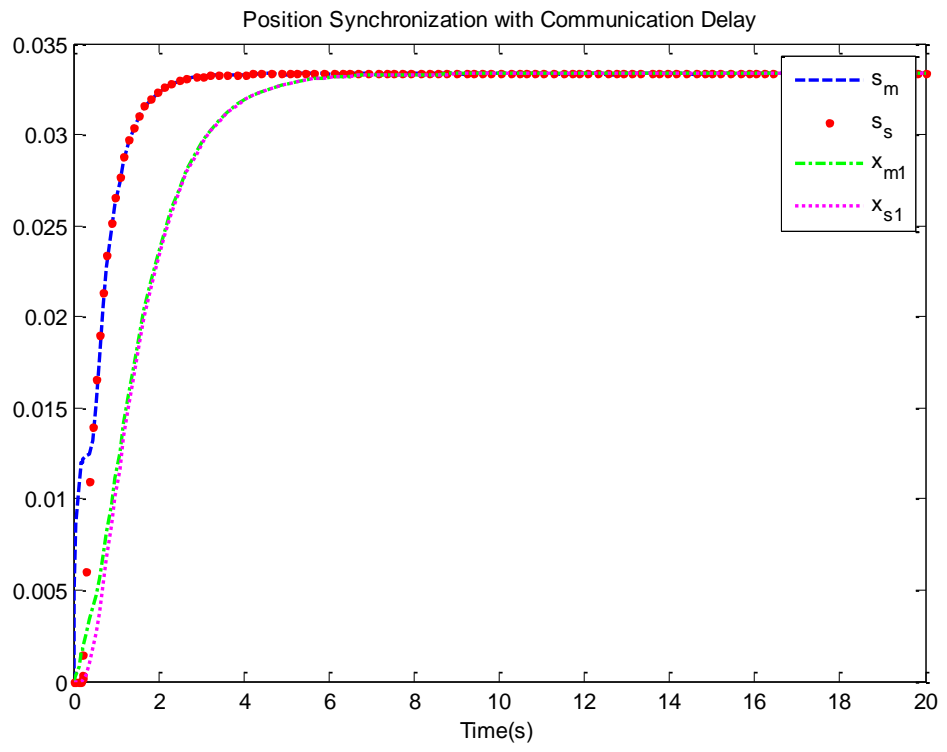

Figure 5

Position synchronization with communication time delay 


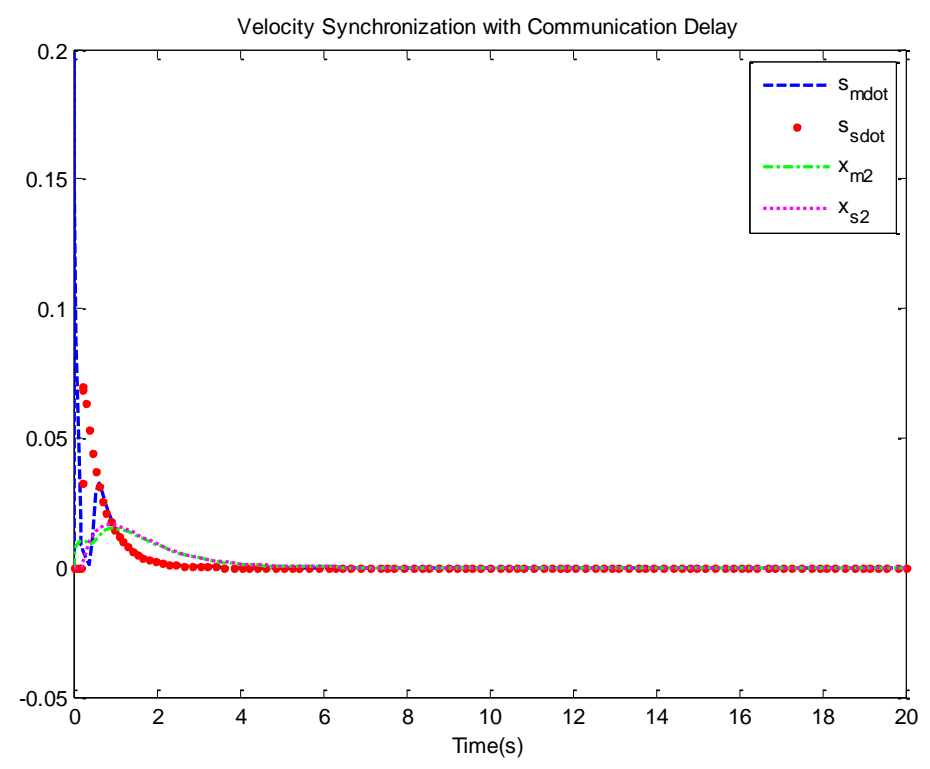

Figure 6

Velocity synchronization with communication time delay

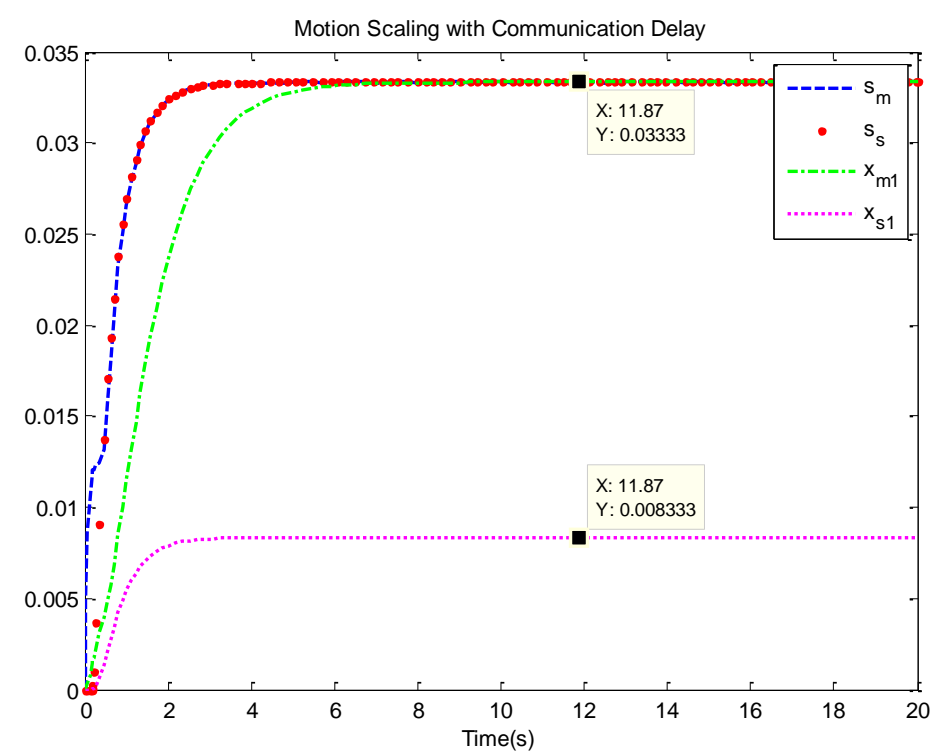

Figure 7

Motion scaling with communication time delay 
We now include some semi-realtime results of the proposed nonlinear controller which are obtained using haptic device in QUARC/Simulink environment. A time-varying operator's force is generated by operating the haptic device along a single axis and trajectories of the resulting master and slave systems are recorded in a time-delayed environment under the control of (29). The results are shown in Figs. 8 and 9. It can be seen that the slave is following the master system and the states finally convergence when the operator's force becomes constant.

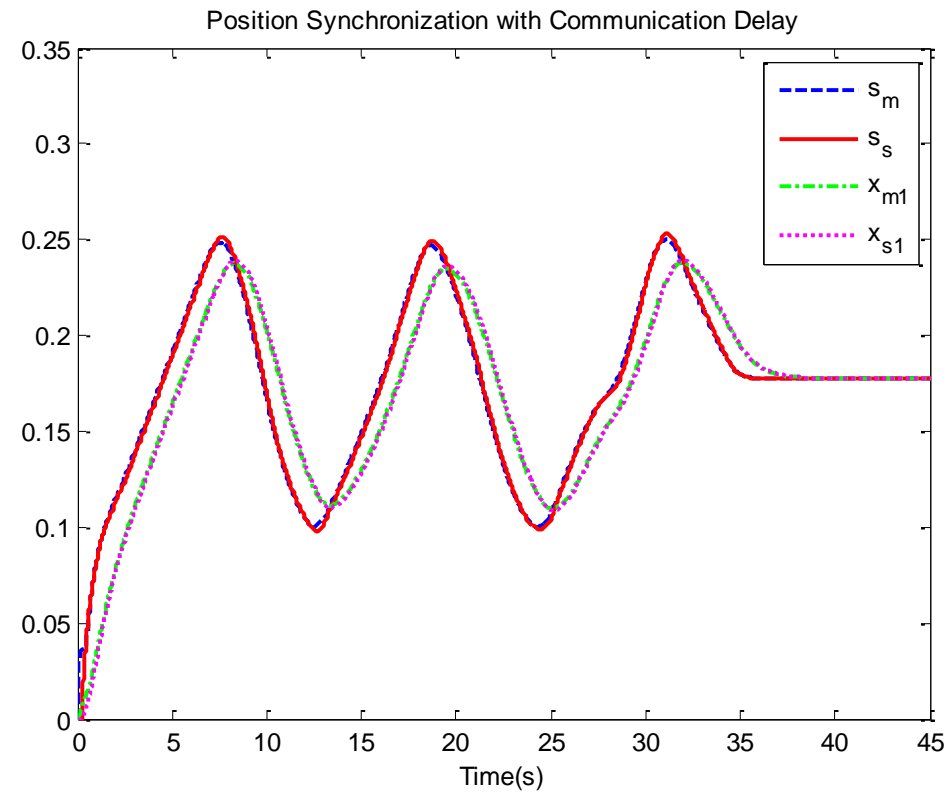

Figure 8

Position synchronization with communication time delay under time varying applied force 


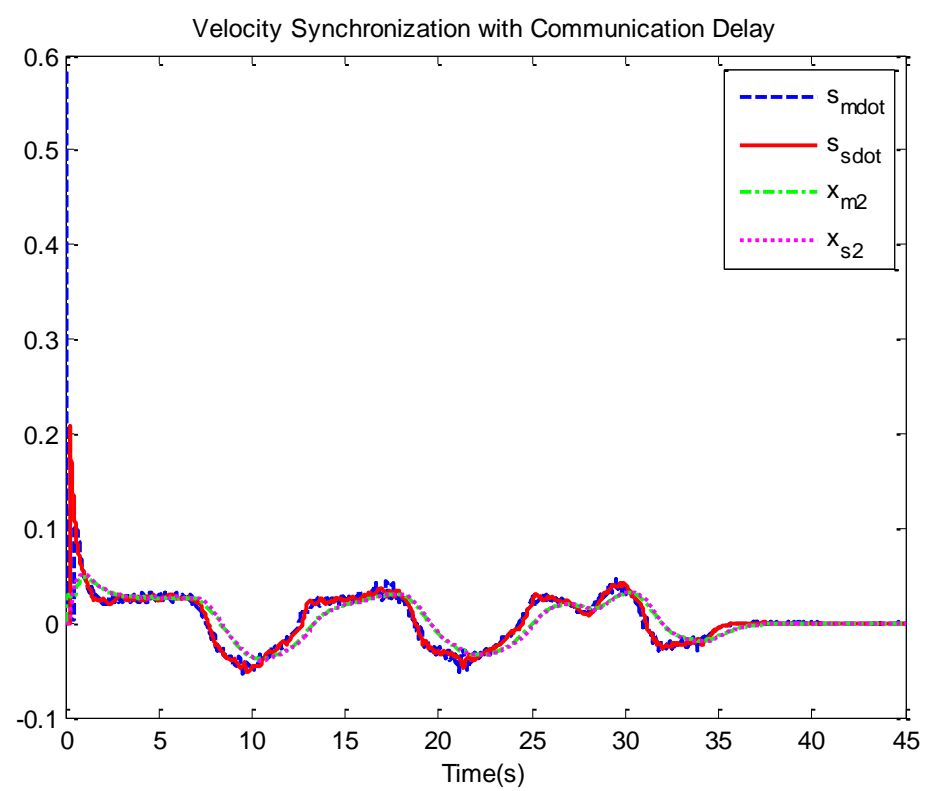

Figure 9

Velocity synchronization with communication time delay under time varying applied force

\section{Conclusions}

This paper has presented the design of a composite state convergence controller for a one degree-of-freedom nonlinear telerobotic system. To deal with the nonlinearity in the master and slave systems, s feedback linearization algorithm is used. The exactly linearized master and slave systems are then used to form the lower complexity composite systems. Through the use of a similarity transformation, a composite slave-error augmented system is constructed. After the composite error is made to evolve as an autonomous, desired behavior is assigned to the telerobotic system. This results in four design conditions which are solved to determine the four unknown control gains. Simulations, as well as semireal time experiments, are finally performed in MATLAB/Simulink environment which shows good performance of the telerobotic system in the absence and presence of communication time delays.

\section{Acknowledgement}

This work was supported by NSERC.

\section{References}

[1] M. Ferre, M. Buss, R. Aracil, C. Melchiorri, C. Balaguer: Advances in Telerobotics, Springer, 2007 
[2] P. F. Hokayem, M. W. Spong: Bilateral Teleoperation: an Historical Survey, Automatica, Vol. 42, 2006, pp. 2035-2057

[3] R. Muradore, P. Fiorini: A Review of Bilateral Teleoperation Algorithms, ACTA Polytechnica Hungarica, Vol. 13, No. 1, 2016, pp. 191-208

[4] J. Artigas, G. Hirzinger: A brief history of DLR's space telerobotics and force feedback teleoperation, ACTA Polytechnica Hungarica, Vol. 13, No. 1, 2016, pp. 239-249

[5] R. Anderson, M. W. Spong: Bilateral Control of Teleoperators with Time Delay: IEEE Transactions on Automatic Control, Vol. 34, No. 5, 1989, pp. 494-501

[6] G. Niemeyer, J. J. E. Slotine: Stable Adaptive Teleoperation, IEEE Journal of Oceanic Engineering, Vol. 16, No. 1, 1991, pp. 152-162

[7] Da Sun, Fazel Naghdy, and Haiping Du: Stability Control of ForceReflected Nonlinear Multilateral Teleoperation System under TimeVarying Delays, Journal of Sensors, vol. 2016, Article ID 4316024, 17 pages, 2016

[8] C. Yang, X. Wang, Z. Li, Y. Li, C. Su: Teleoperation Control Based on Combination of Wave Variable and Neural Networks, IEEE Transactions on Systems, Man, and Cybernetics: Systems, Vol. 47, No. 8, pp. 21252136, Aug. 2017

[9] M. Boukhnifer, A. Ferreira: Hœ Loop Shaping Bilateral Controller for a Two-fingered Tele-micromanipulation System, IEEE Transations on Control Systems Technology, Vol. 15, No. 5, 2007, pp. 891-905

[10] A. Hace, M. Franc: FPGA Implementation of Sliding Mode Control Algorithm for Scaled Bilateral Teleoperation, IEEE Transactions on Industrial Electronics, Vol. 9, No. 3, 2013, pp. 1291-1300

[11] L. Chan, F. Naghdy, D. Stirling: Application of Aadaptive Controllers in Teleoperation Systems, IEEE Transactions on Human-Machine Systems, Vol. 44, No. 3, 2014, pp. 337-352

[12] Y. Yang, C. Hua and X. Guan: Adaptive Fuzzy Finite-Time Coordination Control for Networked Nonlinear Bilateral Teleoperation System, IEEE Transactions on Fuzzy Systems, Vol. 22, No. 3, pp. 631-641, June 2014

[13] A. Suzuki, K. Ohnishi: Frequency Domain Damping Design For Time Delayed Bilateral Teleoperation System Based On Modal Space Analysis, IEEE Transactions on Industrial Electronics, Vol. 60, No. 1, 2013, pp. 177190

[14] J. M. Azorin, O. Reinoso, R. Aracil, M. Ferre: Generalized Control Method by State Convergence of Teleoperation Systems with Time Delay, Automatica, Vol. 40, No. 9, 2004, pp. 1575-1582 
[15] R. Aracil, J. M. Azorin, M. Ferre, C. Pena: Bilateral Control by State Convergence based on Transparency for Systems with Time Delay, Robotics \& Autonomous Systems, Vol. 61, No. 2, 2013, pp. 86-94

[16] J. M. Azorin, R. Aracil, C. Perez, N. M. Garcia, J. M. Sabater: Bilateral Control Architecture for Telerobotics Systems in Unknown Environments, Proc. Euro Haptics, 2008, pp. 13-22

[17] J. M. Azorin, J. A Berna, E. Ianez: Nonlinear Bilateral Control of Teleoperators by State Convergence, Proc. European Control Conference (ECC), Budapest, 2009, pp. 2815-2820

[18] U. Farooq, J. Gu, M. El-Hawary, M. U. Asad, G. Abbas: Fuzzy Model Based Bilateral Control Design of Nonlinear Tele-operation System Using Method of State Convergence, IEEE Access, Vol. 4, 2016, pp. 4119-4135

[19] M. U. Asad, U. Farooq, J. Gu, G. Abbas, R. Liu, V. E. Balas: A Composite State Convergence Scheme for Bilateral Teleoperation Systems, Accepted, IEEE/CAA Journal of Automatica/Sinica, 2019 